Boll. Accademia Gioenia di

Scienze Naturali - Catania
BOLLAG Vol. 52, N. 382 (2019)

Difesa ecosostenibile delle colture agricole pp. DECA29 - DECA36

ISSN 0393-7143

Anno di fondazione 1824

\title{
L'impatto di insetti esotici invasivi negli agro-ecosistemi ${ }^{\dagger}$
}

\author{
Lucia Zappalà $[1]^{*}$ \\ Dipartimento di Agricoltura, Alimentazione e Ambiente, Università degli studi di Catania
}

\begin{abstract}
Riassunto
Le introduzioni accidentali di insetti esotici nei sistemi agricoli e forestali, nonché sulle piante ornamentali anche in ambito urbano, sono aumentate in maniera rilevante in tutto il mondo negli ultimi tre decenni, spesso con gravi conseguenze economiche e ambientali nelle aree recentemente invase. Verranno qui presentati alcuni aspetti di fitofagi di recente o recentissima introduzione in Sicilia. In particolare, saranno prese in esame le strategie di controllo sostenibile di Drosophila suzukii (Moscerino dei piccoli frutti), specie polifaga che attacca frutti a buccia sottile, e l'ancora più polifaga Halyomorpha halys (Cimice asiatica).
\end{abstract}

Parole chiave: Insetti alieni, Drosophila suzukii, Halyomorpha halys, fruttiferi, controllo integrato

\section{Impact of invasive insect pests on agro-ecosystems}

\section{Summary}

The unintentional introductions of exotic insect species in agricultural and forestry systems as well as in urban areas, have been strongly increasing worldwide in the last three decades. This has often caused serious economic and environmental issues in the newly invaded areas. Here a focus on two insect pests recently and very recently introduced in Sicily will be presented. In particular, Drosophila suzukii (Spotted wing Drosophila) affecting the industry of soft-skinned fruits and Halyomorpha halys (Brown stink marmorated bug), extremely polyphagous pest, and their sustainable control strategies will be analyzed.

Key words: Alien species, Drosophila suzukii, Halyomorpha halys, fruit crops, Integrated Pest Management

\section{Introduzione}

L'accidentale introduzione di specie esotiche è un fenomeno in continua crescita in tutto il mondo e tra gli organismi protagonisti di tali processi invasivi, gli insetti si collocano al secondo posto per numerosità dopo le piante vascolari. I nuovi ritrovamenti di specie esotiche di insetti dannosi in agro-ecosistemi e in habitat forestali, nonché a carico del verde urbano e ornamentale in genere, sono pertanto un fenomeno globale in progressiva e rapida crescita, così come lo sono quelle di tutti gli altri organismi esotici, con il 37\% delle nuove segnalazioni registrate negli ultimi 50 anni

\footnotetext{
†Nota presentata nell’Adunanza pubblica del 15 novembre 2018

*e-mail: lzappala@unict.it
} 
[1]. Gli spostamenti di queste specie da un areale all'altro è da correlare per lo più all'azione diretta dell'uomo, vale a dire alle sue attività commerciali, turistiche e al trasporto di materiali di varia natura [2]. Gli effetti dell'introduzione di specie fitofaghe aliene rappresenta un evento più o meno gravemente dannoso per il nuovo areale occupato, tanto dal punto di vista ambientale che economico [3]. Gli inconvenienti del primo tipo sono dovuti al fatto che la nuova entità interagisce abitualmente con altre componenti faunistiche dell'habitat, determinando fenomeni di vicarianza, di competitività ecologica o interferenze di altra natura [4]. In ecosistemi gestiti, tipicamente quelli colturali, si hanno ben noti danni diretti consistenti in perdite quantitative e/o qualitative della produzione, nonché costi aggiuntivi sostenuti per il controllo di tali nuove avversità [5]. Molto spesso, per fronteggiare la dannosità diretta delle specie introdotte si fa ricorso all'impiego di agrofarmaci di sintesi che generalmente causano ulteriori inconvenienti per i loro effetti negativi sia di ordine ecologico che tossicologico sull'uomo e su altri organismi non bersaglio [6]. Tali interventi possono seriamente compromettere programmi di controllo già in atto sulle colture, pur se talora difficilmente messi a punto, improntati al controllo integrato impiegando metodi eco-compatibili o comunque a basso impatto ambientale [7]. Tra gli esempi di nuove introduzioni in Italia di insetti dai negativi effetti sulla gestione di agro-ecosistemi ci sono i due fitofagi polifagi Drosophila suzukii (Matsumura) (Diptera: Drosophilidae) e Halyomorpha halys (Stål) (Hemiptera: Pentatomidae).

\section{Drosophila suzukii (Matsumura)}

È un insetto di origine asiatica che ha invaso la quasi totalità del continente Americano e di quello Europeo nel volgere di pochissimi anni. In Italia, la sua presenza è stata segnalata per la prima volta in Trentino nel 2009 su frutti di mirtillo, fragola e lampone (Fig. 1) [8]. Dopo tale rinvenimento, le segnalazioni nei vari paesi europei e nelle altre regioni italiane comprese quelle più meridionali (in Sicilia nel 2012), si sono susseguite con molta celerità [9].

Le femmine di questa specie, a differenza di quelle degli altri moscerini congeneri, sono dotate di un robusto ovipositore denticolato il quale gli permette di penetrare l'epicarpo e quindi di ovideporre in frutti ancora integri e in corso di maturazione. Tale caratteristica differenzia questa specie da tutte le altre specie di Drosophila che si sviluppano invece soltanto a carico di frutti previamente danneggiati da cause varie, o in fase di decomposizione. Le preferenze di ovidepozione sono positivamente correlate con l'alto tenore zuccherino, valori di $\mathrm{pH}$ non troppo bassi, ridotta consistenza del frutto, bassa densità o assenza di tricomi [10]. Sono suscettibili all'attacco da parte di questo temibile insetto carpofago tutti i frutti a epicarpo sottile, quali lamponi, mirtilli, more, fragole, ciliegie, kiwi, cachi, alcune varietà di uva da tavola e da vino [9]. Il moscerino è in grado inoltre di svilupparsi anche su frutti dall'epicarpo spesso e/o tomentoso (quali mele, pere, melograni, fichi d'India, pesche, agrumi) allorché danneggiati per varie cause (fitofagi, microrganismi fitopatogeni, squilibri fisiologici, grandine) che determinano lesioni sul loro epicarpo [11]. Di notevole importanza ecologica e applicata è inoltre la capacità di questo insetto di ovideporre e svilupparsi a carico di un'ulteriore gamma di frutti spontanei, quali per esempio le bacche di edera, di vischio e di erba morella [12].

Il volo degli adulti e lo sviluppo preimmaginale sono favoriti dai climi miti, mentre temperature superiori ai $30^{\circ} \mathrm{C}$ e soprattutto bassi valori di umidità ne inibiscono l'attività. Queste caratteristiche fanno sì che nelle zone montane gli incrementi delle popolazioni si verifichino a partire dalla tarda primavera fino all'autunno. Nelle zone di pianura viceversa, a causa delle elevate temperature estive, è possibile distinguere due picchi di alta densità di popolazione corrispondenti alla tarda primavera e all'autunno $[10,11,13,14]$. Come conseguenza si possono individuare due periodi in cui l'insetto è presente a bassissime densità, trascorrendo in particolare il periodo invernale da femmina in diapausa riproduttiva [15].

L'individuazione in campo dei frutti attaccati da uova e larve giovani è di difficile attuazione e quindi la valutazione del danno durante la raccolta non sempre risulta agevole. Il monitoraggio delle dinamiche di popolazione può essere facilmente realizzato, grazie all'impiego di trappole 
innescate con aceto di mele, o con vino e aceto, per la cattura degli adulti [16]. Tuttavia, queste sostanze attrattive non sono specifiche per $D$. suzukii e, oltre a catturare anche un cospicuo numero di parassitoidi con conseguenze negative sul controllo naturale della specie [11], le copiose catture indesiderate di altri moscerini possono rendere l'effettiva stima delle popolazioni piuttosto laboriosa. Questo è particolarmente vero nel caso della conta delle femmine, mentre i maschi sono facilmente individuabili grazie a una macchia scura all'apice delle loro ali. La cattura massale con trappole di colore rosso e innescate con i predetti attrattivi evidenzia diversi livelli di efficacia ed è più indicata in condizioni di coltura protetta [16].

Le misure agronomiche per il contenimento di questo insetto passano, ove possibile, attraverso il controllo dell'umidità negli strati più superficiali del suolo atto a condizionare lo sviluppo delle pupe ivi presenti e della chioma, il cui arieggiamento attenua significativamente il volo degli adulti. La disponibilità di frutti suscettibili può essere regolata da raccolte anticipate, tempestive e complete di tutti i frutti coltivati, nonché di quelli spontanei presenti in siepi e bordure adiacenti le colture stesse [16]. Le strategie di controllo chimico, preventivo nei confronti degli adulti e curativo nei confronti degli stadi giovanili nei frutti, sono attualmente limitate a causa di varie difficoltà tecniche quali: (i) la ridotta disponibilità di principi attivi autorizzati per il controllo dell'insetto sulle diverse colture suscettibili; (ii) l'incompatibilità con i tempi di carenza che spesso non permettono un uso appropriato degli insetticidi adoperati considerata la tardiva suscettibilità dei frutti all'attacco del moscerino.

Gli agenti di controllo biologico rinvenuti in associazione a D. suzukii in Italia sono principalmente dei nemici naturali generalisti, quali alcuni predatori tellurici (Coleotteri Stafilinidi e formiche), e due specie di Imenotteri parassitoidi cosmopoliti, Trichopria drosophilae Perkins (Hymenoptera: Diapriidae) (oggi allevato in biofabbriche e reperibile in commercio) (Fig. 1), e Pachycrepoideus vindemiae (Rondani) (Hymenoptera: Pteromalidae), noto anche come iperparassitode [14, 15]. Degna di nota è la pressoché totale mancanza di nemici naturali degli stadi larvali di $D$. suzukii i quali hanno la capacità di resistere, tramite complessi processi immunologici, ai parassitoidi presenti nei nostri ambienti e che attaccano altre specie di drosofile. Tuttavia, nelle aree di origine diverse specie di parassitoidi sono in grado di parassitizzare fino al $18 \%$ delle larve. Alcune di queste specie sono tutt'ora oggetto di valutazione come potenziali candidati agenti di controllo biologico classico eventualmente da importare nei nuovi areali di introduzione di D. suzukii $[17,18]$.

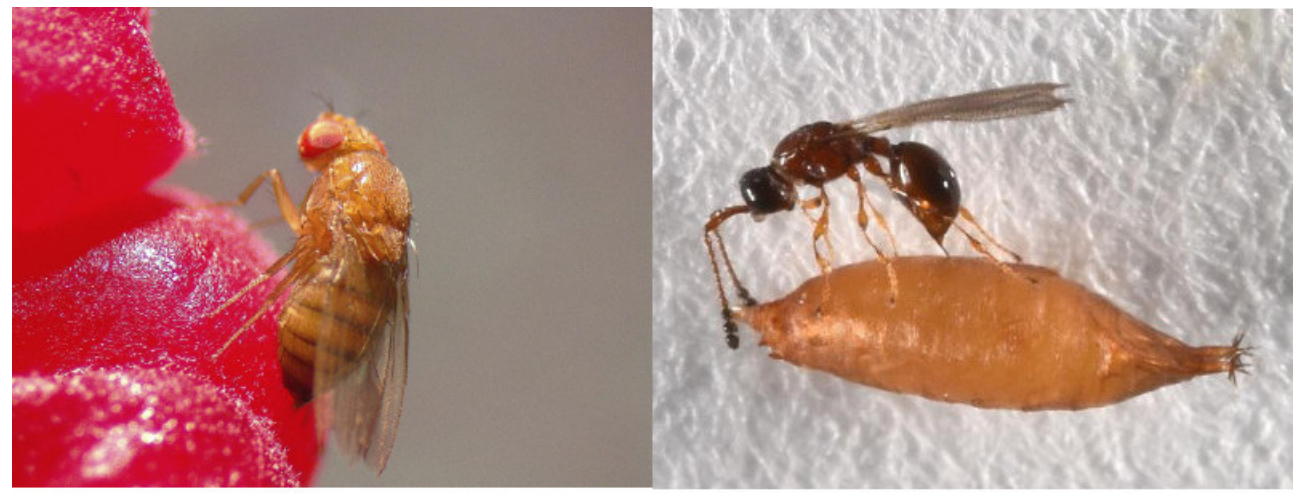

Figura 1: Femmina di Drosophila suzukii su frutto di lampone (sinistra); femmina di Trichopria drosophilae su pupario di D. suzukii (Foto A. Biondi).

\section{Halyomorpha halys (Stål)}

Specie esotica anche questa originaria dell'Asia orientale (Cina, Corea del Sud, Giappone, Taiwan); dal 2010 si è diffusa negli Stati Uniti dove è diventata un importante fitofago di fruttiferi e ortive. Il suo impatto sulle strategie di gestione dei parassiti in Nord America è stato rilevante causando un massiccio aumento nell'uso di pesticidi sintetici anche in quei sistemi agricoli gestiti 
mediante strategie di difesa sostenibile [19]. Negli Stati Uniti l'insediamento e diffusione del fitofago hanno fatto registrare nel 2010 perdite di circa 37 milioni di dollari nei meleti degli Stati atlantici [20]. In Europa, la specie è stata segnalata per la prima volta nel 2004 in Svizzera e si è poi rapidamente diffusa in Bulgaria, Francia, Germania, Grecia, Ungheria, Liechtenstein, Romania, Serbia, Spagna e Russia [21]. In Italia è stata segnalata per la prima volta nel 2012 a Modena [22], ed è ad oggi presente in quasi tutte le regioni inclusa la Sicilia [23]. Dalle informazioni ad oggi disponibili, le uniche regioni italiane in cui ancora la cimice asiatica non è stata segnalata sono Umbria, Molise e Basilicata. Recentemente la specie è stata segnalata in Sud America, in Cile [19]. Sulla base della diffusione globale nel 2016, è stato sviluppato un modello CLIMEX col quale è stato stimato che $H$. halys può diffondersi a livello globale in molte regioni nell'emisfero meridionale in cui vi è un'importante orticoltura [24].

Oltre a produrre danni diretti alle colture, la cimice asiatica è stata segnalata in Asia come vettore di fitoplasmosi per la Paulownia e si sospetta che possa fungere da vettore di fitoplasmi per altre specie vegetali [25]. Inoltre può causare problemi di natura igienico-sanitaria date le aggregazioni che gli adulti producono nelle case e in generale in ambienti antropizzati nel periodo autunnale per svernare cercando rifugio [26]. Ha una vasta gamma di piante ospiti (oltre 170 specie), molte di importanza agraria e/o ornamentale, in particolare tutte le piante da frutto, vite, quasi tutti gli ortaggi, leguminose, cereali (mais, frumento) [19, 21]. È in grado di volare a lunga distanza e di utilizzare piante spontanee per l'alimentazione e la riproduzione [27].

Tanto gli stadi giovanili che gli adulti (Fig. 2) preferiscono nutrirsi di frutti e semi, ma possono attaccare anche tutte le altre parti epigee delle piante ospiti. Le punture di suzione dell'insetto possono portare a lesioni che includono: abscissione, deformità, discromie, cicatrici, depressioni, macchie scure e suberificazioni della polpa dei frutti, con conseguenti gravi perdite economiche [19]. La massima alimentazione da parte degli adulti, si verifica con temperature intorno ai $17^{\circ} \mathrm{C}$, invece cessano di alimentarsi con temperature molto basse intorno ai $3-6^{\circ} \mathrm{C}$ e molto alte al di sopra dei $29^{\circ} \mathrm{C}$ [27].

Alle prime forti infestazioni della cimice asiatica verificatesi negli Stati Uniti nel 2010, le uniche indicazioni per la gestione del fitofago nei diversi sistemi colturali, riguardavano applicazioni di insetticidi. Tuttavia, molti frutticoltori che già applicavano prodotti autorizzati per le specie di cimici indigene, hanno riportato livelli di efficacia molto ridotti nei confronti di questo pentatomide esotico. Inoltre, l'aumento delle applicazioni insetticide ad ampio spettro di azione ha portato ad infestazioni di fitofagi secondari, solitamente controllati efficacemente dai nemici naturali [20]. Studi condotti su popolazioni asiatiche, hanno dimostrato che $H$. halys è attratta dal feromone methyl (2E,4E,6Z)-decatrienoate (MDT), feromone di aggregazione della cimice verde dalle ali marroni, Plautia stali Scott. L'utilizzo di questo feromone però ha mostrato alcuni limiti applicativi: gli adulti della cimice asiatica non sono attratti dalle trappole innescate con tale feromone nelle fasi di innalzamento delle popolazioni, mentre lo sono le ninfe [28].

Dopo alcuni anni è stato identificato il feromone di aggregazione prodotto dalla cimice asiatica [29] e le trappole innescate con questo feromone in combinazione con MDT, hanno fatto registrare una risposta attrattiva sinergica nei confronti di adulti e ninfe [30].

Il comportamento altamente polifago e vagile di tale insetto è stato sfruttato provando ad utilizzare, come tecnica di gestione, colture trappola, come il sorgo e il girasole, in quanto sono state identificate tra le più attrattive [31]. Altre tecniche di controllo sostenibile prevedono l'impiego di mezzi meccanici e agronomici, quali ad esempio la rimozione meccanica delle ovature; l'insacchettamento individuale dei frutti; il controllo di ospiti alternativi; la creazione di rifugi trappola per lo svernamento e infine l'uso di reti multifunzionali (antigrandine e anti insetto) situate al disopra delle piante per evitare l'accesso alla cimice asiatica. Dai primi risultati è stato riscontrato un buon controllo di $H$. halys attraverso l'utilizzo di queste reti che hanno permesso di ridurre le applicazioni insetticide [32].

Per quanto concerne il controllo biologico della specie, le prime indagini condotte negli Stati Uniti hanno evidenziato la presenza di potenziali nemici naturali per la cimice asiatica, con tasso in percentuale che va da $<1 \%$ al 55,3\% [33]. In Europa, studi di laboratorio e di campo hanno 
documentato che dal $31 \%$ al $62 \%$ delle ovature di $H$. halys sono state attaccate da predatori generalisti [34]. I più importanti nemici naturali nativi di $H$. halys più importanti, riscontrati negli Stati Uniti, sono imenotteri parassitoidi oofagi [35], anche se con percentuali molto basse di parassitismo. Sulla base di dati di campo raccolti in Nord America e in Europa, risulta che sono stati isolati da uova della cimice asiatica, principalmente parassitoidi appartenenti alle famiglie degli Scelionidae, Eupelmidae e Encyrtidae [36]. Tuttavia il grado di parassitismo delle uova non è elevato ed ha un impatto molto limitato [33]. Data la scarsa efficienza dei nemici naturali endemici di H. halys, sono stati avviati negli Stati Uniti programmi di controllo biologico classico [37]. Dal 2007, Trissolcus japonicus e altre specie appartenenti allo stesso genere, sono stati mantenuti in strutture di quarantena negli Stati Uniti per valutare il loro ruolo come agenti di controllo biologico classico [37] e nel 2015 la presenza del primo è stata notata in campo [38]. Nel 2017 T. japonicus è stato ritrovato accidentalmente in diverse località del Canton Ticino (Svizzera) [39] e in Lombardia nell'agosto del 2018 [40]. Tali ritrovamenti apriranno senz' altro nuovi e interessanti scenari nel controllo sostenibile di questa specie con particolare riferimento al controllo biologico a mezzo di parassitoidi esotici.

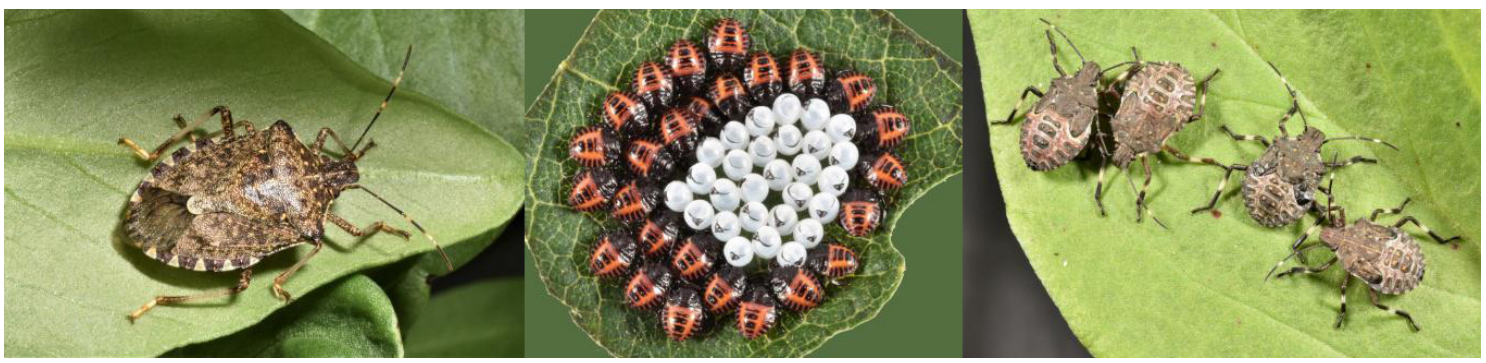

Figura 2: Halyomorpha halys: adulto (sinistra); uova appena schiuse e neanidi neonate (centro); ninfe (destra)

(Foto G. Siscaro).

\section{Considerazioni conclusive}

I problemi entomologici di maggiore rilevanza tecnico-economica in Italia, come in altri paesi del mondo, sono legati il più delle volte all'introduzione di fitofagi di provenienza esotica. Come ben noto, questi fenomeni di invasione da parte di dannosi organismi alloctoni si collegano a molteplici cause tra cui un ruolo chiave svolgono gli intensi flussi commerciali e il cambiamento climatico. In tale contesto, va considerato che la strategia che in numerose occasioni si è rivelata vincente, è l'introduzione di agenti ausiliari (entomofagi, entomopatogeni) dagli stessi luoghi di origine delle specie nocive (controllo biologico classico). Tuttavia, la normativa vigente limita fortemente tali iniziative; risulta pertanto auspicabile una revisione di tali posizioni, anche alla luce delle indicazioni contenute nel documento di recente pubblicazione da parte della European and Mediterranean Plant Protection Organization (EPPO) [41], che fornisce ai singoli stati i protocolli per la valutazione e validazione degli agenti biotici da introdurre in termini sia di effetti target che non-target. Il controllo biologico classico può certamente rappresentare uno strumento essenziale al fine di contenere il più possibile l'impiego di prodotti fitosanitari nei programmi di controllo delle specie invasive.

\section{Riferimenti bibliografici}

Seebens, H., Blackburn, T.M., Dyer, E.E., Genovesi, P., Hulme, P.E., Jeschke, J.M., Pagad, S., Pyšek, P., Winter, M., Arianoutsou, M., Bacher, S., Blasius, B., Brundu, G., Capinha, C., Celesti-Grapow, L., Dawson, W., Dullinger, S., Fuentes, N., Jäger, H., Kartesz, J., Kenis, M., Kreft, H., Kühn, I., Lenzner, B., Liebhold, A., Mosena, A., Moser, D., Nishino, M., Pearman, D., Pergl, J., Rabitsch, W., Rojas-Sandoval, J., Roques, A., Rorke, S., Rossinelli, S., Roy, H.E., Scalera, R., Schindler, S., Štajerová, K., Tokarska-Guzik, B., Van Kleunen, M., Walker, K., 
Weigelt, P., Yamanaka, T., Essl, F. 2017, No saturation in the accumulation of alien species worldwide. Nat. Commun. 8, 14435.

Barbagallo, S., Colombo, M., La Pergola, A., Zappalà, L. 2009, Problemi fitoiatrici e ambientali connessi all'introduzione di fitofagi e fitomizi alieni. Protezione delle Colture, 2, 34-38.

Pimentel, D., Lach, L., Zuniga, R., Morrison, D. 2000, Environmental and economic costs of nonindigenous species in the United States. BioScience 50, 53-65.

Clavero, M., García-Berthou, E. 2005, Invasive species are a leading cause of animal extinctions. Trends Ecol. Evol., 20,110.

Ragsdale, D.W., Landis, D.A., Brodeur, J., Heimpel, G.E., Desneux, N. 2011, Ecology and management of the soybean aphid in North America. Annu. Rev. Entomol. 56, 375-399.

Desneux, N., Decourtye, A., Delpuech, J.M. 2007, The sublethal effects of pesticides on beneficial arthropods. Annu. Rev. Entomol., 52, 81-106.

Biondi, A., Guedes, R.N.C., Wan, F.H., Desneux, N. 2018, Ecology, Worldwide Spread and Management of the Invasive South American Tomato Pinworm, Tuta absoluta: Past, Present, and Future. Annu. Rev. Entomol. 63, 239-258.

Cini, A., Ioriatti, C., Anfora, G. 2012, A review of the invasion of Drosophila suzukii in Europe and a draft research agenda for integrated pest management. Bulletin of Insectology, 65, 149-160.

Asplen, M.K., Anfora, G., Biondi, A., Choi, D.-S., Chu, D., Daane, K.M., Gibert, P., Gutierrez, A.P., Hoelmer, K.A., Hutchison, W.D., Isaacs, R., Jiang, Z.-L., Kárpáti, Z., Kimura, M.T., Pascual, M., Philips, C.R., Plantamp, C., Ponti, L., Vétek, G., Vogt, H., Walton, V.M., Yu, Y., Zappalà, L., Desneux, N. 2015, Invasion biology of spotted wing Drosophila (Drosophila suzukii): a global perspective and future priorities. J. Pest Sci., 88, 469-494.

Arnó, J., Solà, M., Riudavets, J., Gabarra, R. 2016, Population dynamics, non-crop hosts, and fruit susceptibility of Drosophila suzukii in Northeast Spain. J. Pest Sci., 89, 713-723.

Wang, X.G., Stewart, T.J., Biondi, A., Chavez, B.A., et al. 2016 Population dynamics and ecology of Drosophila suzukii in Central California. J. Pest Sci., 89, 701-712.

Kenis, M., Tonina, L., Eschen, R., van der Sluis, B., Sancassani, M., Mori, N., Haye, T., Helsenet, H. 2016, Non-crop plants used as hosts by Drosophila suzukii in Europe. J. Pest Sci. 89, 735748.

Mazzetto, F., Pansa, M.G., Ingegno, B.L., Tavella, L., Alma, A. 2015, Monitoring of the exotic fly Drosophila suzukii in stone, pome and soft fruit orchards in NW Italy. J. Asia-Pac. Entomol. 18, 321-329.

Gugliuzzo, A., Cavallaro, C., Alinç, T., Finocchiaro, E., Tropea Garzia, G., Siscaro G., Zappalà L., Biondi A. 2018, Population dynamics of Drosophila suzukii and first data on its parasitoids on Mount Etna (Italy). Book of Abstract of the XI European Congress of Entomology, 2-6 July 2018, Napoli, 235.

Rossi-Stacconi, M.V., Buffington, M., Daane, K.M., Dalton, D.T., Grassi, A., Kaçar, G., Miller, B., Miller, J.C., Baser, N., Ioriatti, C., Walton, V.M., Wiman, N.G., Wang, X., Anfora, G. 2015, Host stage preference, efficacy and fecundity of parasitoids attacking Drosophila suzukii in newly invaded areas. Biol. Control 84, 28-35.

Haye, T., Girod, P., Cuthbertson, A.G.S., Wang, X.G., Daane, K.M., Hoelmer, K.A., Baroffio, C., Zhang, J.P., Desneux N. 2016, Current SWD IPM tactics and their practical implementation in fruit crops across different regions around the world. J. Pest Sci. 89, 643-651. 
Daane, K.M., Wang, X.G., Biondi, A., Miller, B., Miller, J.C., Riedl, H., Shearer, P.W., Guerrieri, E., Giorgini, M., Buffington, M., van Achterberg, K., Song, Y., Kang, T., Yi, H., Jung, C., Lee, D.W., Chung, B.-K., Hoelmer, K.A., Walton, V.M. 2016, First foreign exploration for Asian parasitoids of Drosophila suzukii. J. Pest Sci. 89, 823-835.

Biondi, A., Wang, X.G., Miller, J.C., Miller, B., Shearer, P.W., Zappalà, L., Siscaro G., Walton V. W., Hoelmer K. A., Daane K.M. 2017, Innate olfactory responses of Asobara japonica (Hymenoptera: Braconidae) toward fruits infested by the invasive spotted wing Drosophila. J. Insect behav. 30, 495-506.

Leskey, T.C., Nielsen, A.L. 2018, Impact of the Invasive Brown Marmorated Stink Bug in North America and Europe: History, Biology, Ecology, and Management. Annu. Rev. Entomol. 63, 599-618.

Leskey, T.C., Short, B.D., Butler, B.R., Wright, S.E. 2012, Impact of the Invasive Brown Marmorated Stink Bug, Halyomorpha halys (Stål), in Mid-Atlantic Tree Fruit Orchards in the United States: Case Studies of Commercial Management. Psyche: A Journal of Entomology, Article ID 535062, 1-14.

Haye, T., Gariepy, T., Hoelmer, K., Rossi, J.P., Streito, J.C., Tassus, X., Desneux, N. 2015, Range expansion of the invasive brown marmorated stinkbug, Halyomorpha halys: an increasing threat to field, fruit and vegetable crops worldwide. J. Pest. Sci. 88, 665-673.

Maistrello, L., Dioli, P., Vaccari, G., Nannini, R., Bortolotti, P., Caruso, S., Costi, E., Montermini, A., Casoli, L., Bariselli, M. 2014, Primi rinvenimenti in Italia della cimice esotica Halyomorpha halys, una nuova minaccia per la frutticoltura. ATTI Giornate Fitopatologiche, 1, 283-288.

Caparezza, A., Lo Verde, G. 2017, First record Halyomorpha halys (Stål, 1885) (Hemiptera Peantatomidae) in Sicily. Naturalista Siciliano 61, 139-145.

Kriticos, D.J., Kean, J.M., Phillips, C.B., Senay, S., Acosta, H., Haye, T. 2017, The potential global distribution of the brown marmorated stink bug, Halyomorpha halys Stål (Hemiptera: Pentatomidae): a critical threat to plant biodiversity. J. Pest Sci., 90, 1033-1043.

Jones, J.R., Lambdin, P.L. 2009, New county and state records for Tennessee of an exotic pest, Halyomorpha halys (Hemiptera: Pentatomidae), with potential economic and ecological implications. Fla. Entomol. 92, 177-179.

Lee, D.H., Short, B.D., Joseph, S.V., Bergh, J.C., Leskey, T.C. 2013, Review of the biology, ecology, and management of Halyomorpha halys (Hemiptera: Pentatomidae) in China, Japan, and the Republic of Korea. Environ. Entomol., 42, 627-641.

Wiman, N.G., Walton, V.M., Shearer, P.W., Rondon, S.I., Lee, J.C. 2014, Factors affecting flight capacity of brown marmorated stink bug (Hemiptera: Pentatomidae). J. Pest Sci. 88, 37-47.

Morrison, W.R., Lee, D.H., Short, B.D., Khrimian, A., Leskey, T.C. 2016, Establishing the behavioral basis for an attract-and-kill strategy to manage the invasive Halyomorpha halys in apple orchards. J. Pest Sci. 89, 81-96.

Khrimian, A., Zhang, A., Weber, D.C., Ho, H.Y., Aldrich, J.R., Vermillion, K.E., Siegler M.A., Shirali S., Guzman, F., Leskey, T.C. 2014, Discovery of the aggregation pheromone of the brown marmorated stink bug (Halyomorpha halys) through the creation of stereoisomeric libraries of 1-bisabolen-3-ols. J. Nat. Products, 77, 1708-1717.

Weber, D.C., Leskey, T.C., Walsh, G.C., Khrimian, A. 2014, Synergy of aggregation pheromone with methyl (E, E, Z)-2, 4, 6-decatrienoate (Hemiptera: Pentatomidae). In attraction of Halyomorpha halys. J. Econ. Entomol. 107, 1061-1068. 
Owens, D.R., Herbert Jr, D.A., Dively, G.P., Reisig, D.D., Kuhar, T.P. 2013, Does feeding by Halyomorpha halys (Hemiptera: Pentatomidae) reduce soybean seed quality and yield? J. Econ. Entomol. 106, 1317-1323.

Candian, V., Pansa, M. G., Briano, R., Peano, C., Tedeschi, R., \& Tavella, L. 2018, Exclusion nets: a promising tool to prevent Halyomorpha halys from damaging nectarines and apples in NW Italy. Bulletin of Insectology 71, 21-30.

Cornelius, M.L., Dieckhoff, C., Hoelmer, K.A., Olsen, R.T., Weber, D.C., Herlihya M.V., Talamasd E.J., Vinyarde B.T., Greenstoneet M.H. 2016, Biological control of sentinel egg masses of the exotic invasive stink bug Halyomorpha halys (Stål) in Mid-Atlantic USA ornamental landscapes. Biol. control 103, 11-20. 